\title{
Downscaling climate model outputs into local and regional weather elements by classification and regression
}

\author{
W. Enke*, A. Spekat \\ Institute of Meteorology, Free University of Berlin, C.-H.-Becker-Weg 6-10, D-12165 Berlin, Germany
}

\begin{abstract}
A 2-step downscaling method for general circulation model (GCM) outputs is presented. The investigation is based on daily upper air geopotential and humidity fields (Atlantic-European sector) and surface observations (52 German climate stations) from 1966 to 1993. In the first step, significant circulation patterns are identified using cluster analysis which takes advantage of information exclusively from upper air fields. This leads to composite charts which can be readily interpreted synoptically, but which only explain a portion of the variance of the local weather elements. In the second step, a conditional (weather pattern-dependent) stepwise screening regression analysis is performed for each weather element and 6 German climate regions. A principal finding is that the modelled (downscaled) local climate is in good agreement with observations, particularly for the temperature regime, due to the fact that a major part of the variance is explained after the 2 steps. Including $700 \mathrm{hPa}$ humidity slightly improves the explained variance. An application to a GCM control run is added. It shows that the method is capable of reconstructing interannual variability of local weather elements.
\end{abstract}

KEY WORDS: Climate modelling - Statistical downscaling - Cluster analysis - Regression analysis . General circulation models

\section{INTRODUCTION}

Even though the capabilities of global climate models have progressed considerably, the accuracy of their predictions is still in a stage of uncertainty. This is definitely the case with respect to regional climate modelling. However, data on weather elements with a high spatial and temporal resolution are in great demand, e.g. for climate impact modellers (Enke 1994). The typical spatial range of state-of-the-art general circulation models (GCMs), which are still the only source for long-term simulation, is a few hundred $\mathrm{km}$ or more (e.g. T42 resolution $\approx 250 \mathrm{~km}$ horizontal resolution). To overcome this gap, 3 strategies have been developed: (1) nested high resolution regional climate models (Giorgi 1990), (2) statistical-dynamical time-slice modelling, which aims at a coupling of different scales by using high resolution dynamic models and derived statistical relationships, e.g. from large-scale weather patterns (Frey-Buness et al. 1995), and (3) pure statistical

\footnotetext{
-E-mail: wenke@bibo.met.fu-berlin.de
}

methods. Basically, statistical downscaling inverts parameterizing procedures used in numerical weather prediction models. Dynamical models have incorporated schemes to estimate processes below the scale of the model which cannot be modelled directly. Statistical downscaling, in turn, uses large-scale model outputs to estimate these subscale processes. For downscaling, only a 'Perfect Prog' approach (Klein et al. 1959) is possible, because of the lack of temporal relationships between climate model outputs and observed weather elements (von Storch 1995). The most common statistical downscaling methods use Multiple Regression Analysis (Klein et al. 1959), Empirical Orthogonal Functions (EOFs) (Kutzbach 1967), Fuzzy Clustering (Bardossy et al. 1995), and Classification and Regression Trees (CART) (Burrows et al. 1995). For a comprehensive review, see Giorgi \& Mearns (1991) and Zorita et al. (1995).

All statistical methods share the benefit of being cost-efficient with respect to use of CPU time, in comparison to statistical-dynamical or pure dynamical methods. 
It is evident that a combination of classification and post-processing (e.g. regression) is a beneficial downscaling approach. Computing EOFs would be a possible, though less suitable, alternative. However, the first few EOFs which may be interpreted synoptically describe only the principal part of the explainable variance. The difficulty in interpreting EOFs increases with respect to their rank. Furthermore, if heterogeneous fields, such as thickness, geopotential height and humidity, are used together, extra care in interpretation must be taken (Chen \& Harr 1992). CART, on the other hand, has come into use recently, and is an example of a hierarchical classification method which may not necessarily lead to a global structural optimum. However, unwanted subjectiveness is introduced due to 2 facts: (1) CART builds the classification structure using knowledge about the predictand, whereas methods like EOFs or cluster analysis group patterns per se; (2) CART requires a prescribed threshold in each level to binarize the predictand, but establishing this threshold is complicated. Recent results have shown the validity of CART (Zorita et al. 1995), although a combination of neural nets and CART may be more appropriate (Jessup \& Burrows 1996).

Note: In the past, classification has been done subjectively (Lamb 1953, Hess \& Brezowsky 1969). A review of other subjective classification methods is given in Lamb (1972, p 267-269). It must be noted that objective methods are much more flexible and generally superior.

The method described here uses a modelling approach of classification and multiple regression that has been applied operationally by the German Weather Service for nearly $30 \mathrm{yr}$. Clustering, which is adapted to this downscaling approach, generates composites that can readily be interpreted synoptically, and has the advantage that each day is categorized in an explicit way. It is evident that the same effects (e.g. warm or wet days) may be the result of different weather types; thus for each class (or weather pattern) a separate regression analysis must be performed.

\section{DATA}

The classification step requires daily grid fields. For our investigation we used 28 years (1966 to 1993) of geopotential heights of the 1000,700 , and $500 \mathrm{hPa}$ levels and the 700-1000, 500-1000 and 500-700 hPa thicknesses in the North Atlantic-European sector $\left(40^{\circ}\right.$ to $60^{\circ} \mathrm{N}, 10^{\circ} \mathrm{W}$ to $20^{\circ} \mathrm{E}$, resolution $5^{\circ} \times 5^{\circ}$; see Fig. 1) from the German Weather Service.

The regression step requires time series of daily surface data. This investigation uses observations at 52 German climate stations obtained from the German

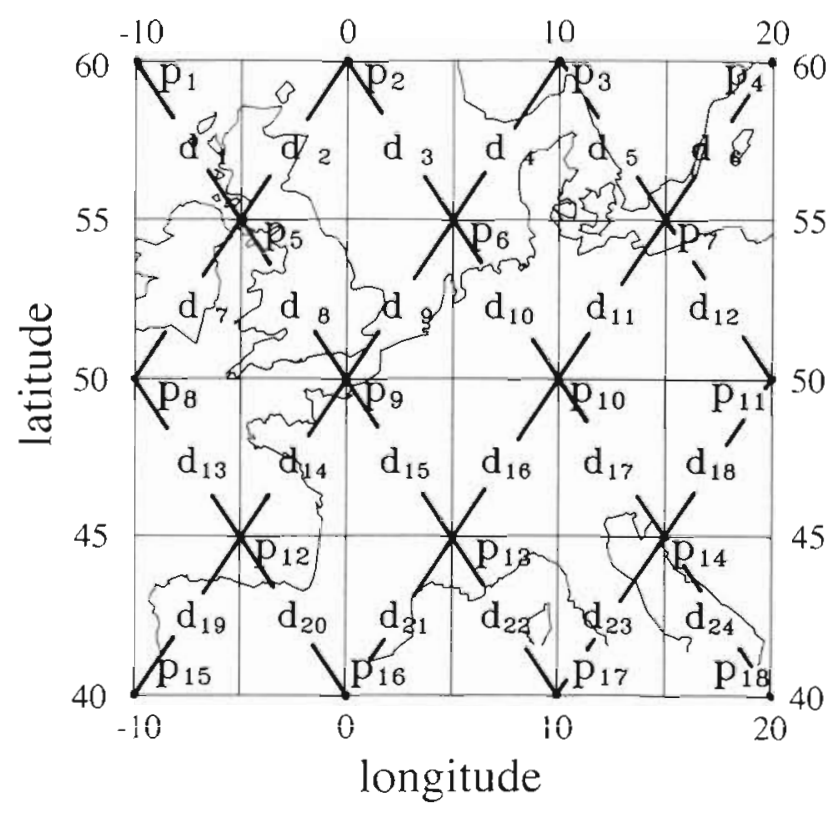

Fig. $1.5^{\circ} \times 5^{\circ}$ gridnet over Western and Central Europe and location of horizontal differences $\mathrm{d}_{1} \ldots \mathrm{d}_{24}$ (see $\mathrm{Eq}$. 1) used for this study. The points $p_{1}$ to $p_{: 8}$ were used to define predictors for the screening regression analysis (see Table 1)

Weather Service, containing the following daily weather elements/predictands:

- Tmax: maximum temperature $(\mathrm{K})$

- Tmin: minimum temperature (K)

- Tmean: mean temperature $(\mathrm{K})$

- RH: mean relative humidity (\%)

- Cloud: mean cloud cover (octas)

- Sun: absolute sunshine duration (h)

- Prec: sum of precipitation $(\mathrm{mm})$; also computed as probability of precipitation over given thresholds

Both the actual daily values and deviations from the annual cycle were used. In order to derive the annual cycle, daily means of each weather element for the period 1951 to 1994 were calculated. A running $17 \mathrm{~d}$ mean was applied to smooth short-term fluctuations caused by the limited data sample. However, for the downscaling procedure only those 28 years for which upper air data were available (1966 to 1993) could be used. A better areal representation is achieved by grouping the stations into 6 major climate zones (E. Hollan pers. comm.): (1) coast, (2) lowlands, (3) elevated areas below $500 \mathrm{~m}$, (4) elevated areas between 500 and $1000 \mathrm{~m},(5)$ alpine area above $1000 \mathrm{~m}$ and (6) upper Rhine Valley (cf. Fig. 2).

Potential predictors (cf. Table 1) for the screening regression analysis (cf. Section 3.2) are exclusively derived from upper air geopotential fields. Further potential predictors are pseudo-persistence (cf. note in Section 3.2) and the Julian day of the year. 


\section{METHOD}

Thorough studies in the field of statistical weather forecasting (Enke 1986, Balzer 1994) have proved that a conditional, linear, multiple regression analysis is appropriate for statistical downscaling and forecasting purposes. Independent variables/predictors and coefficients are optimized using weather patterns in consideration of statistical stability. Conditionality is incorporated by classifying all data in 2 steps (Mucha 1992). The data sample is divided into 4 seasons, i.e. DJF, MAM, JJA, SON.

\subsection{Classification}

In this study, a modified minimum distance method is used, which is a type of partitioning cluster analysis (Forgy 1965). It is based on a starting partition for a subjectively prescribed number of classes, followed by the assignment of all remaining weather patterns to their most similar class, then rearranging weather patterns iteratively to reach a stable final state.

\subsubsection{Starting partition}

A starting partition of objects/weather patterns with 'maximum dissimilarity' is determined by first selecting (subjectively) an initial weather situation $\mathrm{O}_{1}$. In a stepwise procedure, the most dissimilar weather patterns $\mathrm{O}_{2} \ldots \mathrm{O}_{n}$ are selected from the archive of daily grid fields (cf. Fig. 3 , objects marked ' $a$ '). To assign weather patterns to a cluster the normalized euclidean distance measure $D$ (Eq. 1) is used. By aid of this normalization, several types of fields (topographies, humidity, etc.) can be incorporated.

$$
D=\frac{\sum_{i=1}^{m}\left(\left|d p_{i}-d_{i}\right| \cdot \alpha_{1}\right)}{\sum_{i=1}^{m} \max \left(\left.\left|d p_{i}\right|\right|_{i}\left|d_{i}\right|\right)}
$$

where $d p_{i}=$ horizontal differences of a given field (e.g. mean weather pattern or class mean); $d_{1}=$ horizontal differences of the field to compare (e.g. daily weather pattern); $\alpha=$ weights of the differences, depending on the distance from the central ref-

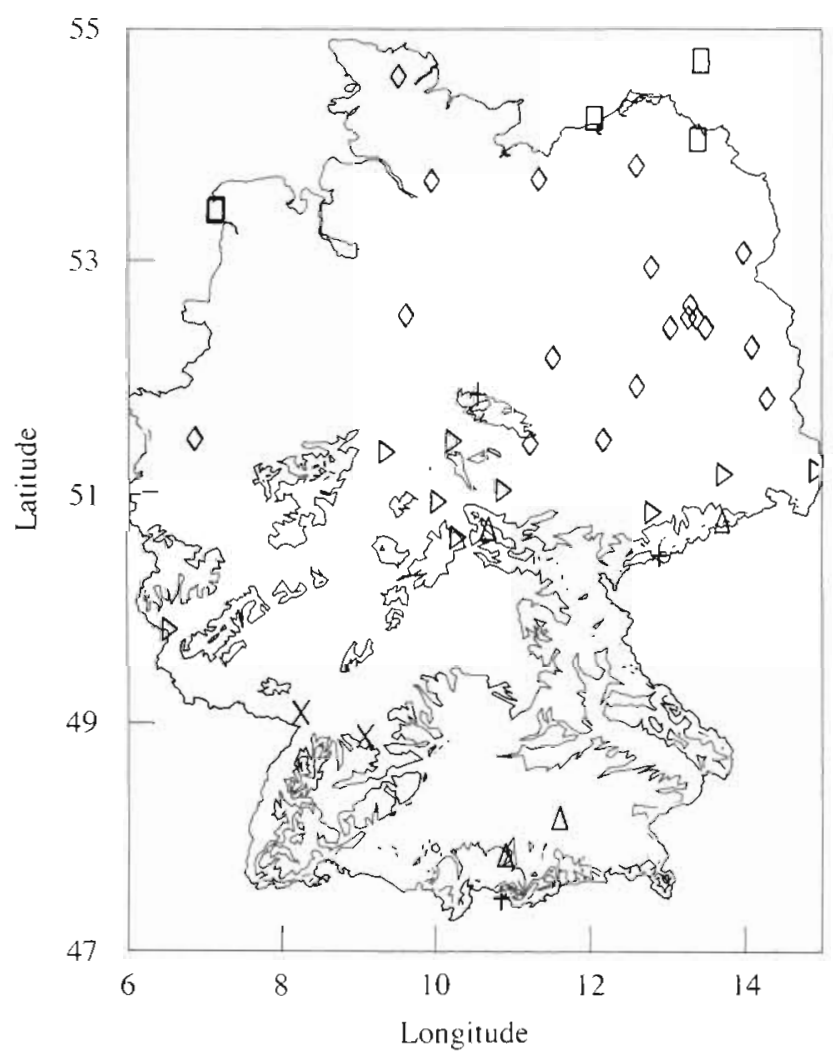

Fig. 2. Distribution of meteorological observation stations in the climate zones in Germany. $(\square)$ Coastal, $(\diamond)$ lowlands, $(\triangleright)$ elevation $<500 \mathrm{~m},(\Delta)$ elevation $<1000 \mathrm{~m},(+)$ mountains $>1000 \mathrm{~m},(\times)$ upper Rhine Valley

Table 1. Potential predictors submitted to the screening regression analysis For numerical indices of p, see Fig. 1

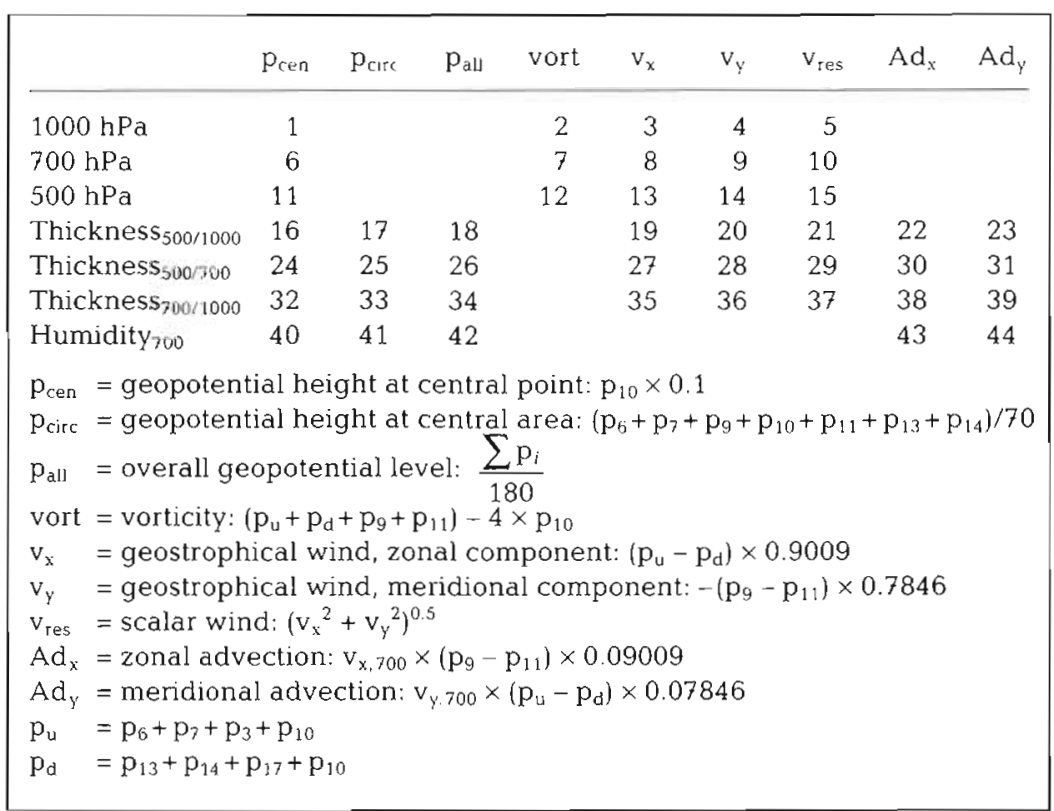




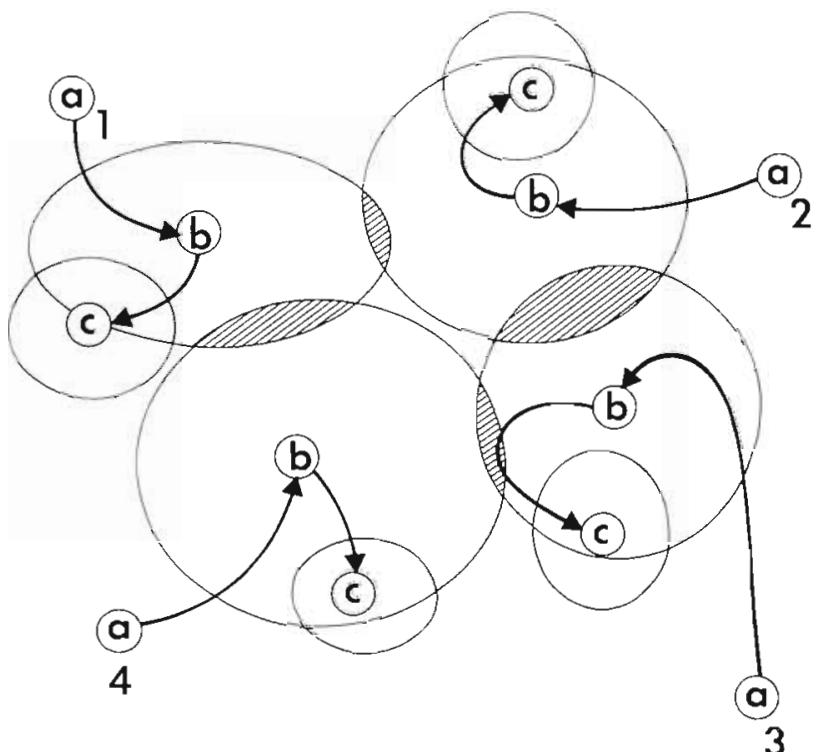

Fig. 3. Schematic representation of the 3 classification steps. Note the shift in centroid position and the variance-reducing effect of iteratively exchanging objects

erence point (see Fig. 1); $m=$ number of horizontal differences (see Fig. 1).

Empirically, it was found that the following combination of fields should be used to compute $D_{\text {total }}$ :

$D_{\text {total }}=D_{1000, Y}+D_{500 / 1000, Y}+D_{500 / 1000, Y}+D_{1000, \mathrm{t}}+D_{500 / 1000, \mathrm{t}}$

where the indicies mean: 1000, absolute topography $1000 \mathrm{hPa}$; $500 / 1000$, thickness $500-1000 \mathrm{hPa}$; y, yesterday, i.e. day-1; $t$, today.

To account for the processual character of the weather, the computation of $D$ is based on fields from 2 time steps, $h$ (00 UTC) and $h+24$.

To obtain the other members of the starting partition a set of the most dissimilar weather situations is computed as follows:

$$
D_{\text {total }}(w)=\sum_{k=1}^{n} D_{\text {totid }}(k) \Rightarrow \operatorname{Max}
$$

where $w=1, \ldots, m_{i} m=$ number of days; $n=2, \ldots, 10$ (number of classes)

Additional effects caused by the (areal) mean level of the selected patterns are dealt with in the regression step (Section 3.2).

\subsubsection{Assignment}

With 10 weather patterns used as 'condensation nuclei', i.e the starting partition, all remaining objects' weather patterns are then assigned to their most similar classes.

$$
D_{\text {total }}(k) \Rightarrow \operatorname{Min}
$$

where $k=1, \ldots, n ; n=$ number of classes.

In this phase the class centroids shift with each newly 'absorbed' member. Consequently the multidimensional distance between the class centroids continues to decrease, but the variability within the individual classes increases (cf. Fig. 3, objects marked ' $b$ '). In this stage, the assignment of individual weather patterns may be ambiguous because of overlapping clusters (see Fig. 3). Therefore a further sub-step is needed.

\subsubsection{Exchange}

We optimize the class assignment by iteratively exchanging objects-using the minimum distance method - until a stable state is reached. This results in 2 benefits: the centroids are better separated, and their internal variability is reduced (cf. Fig. 3, objects marked ' $C$ '). As a result of the sub-steps in Sections 3.1.1 to 3.1.3, averaged weather patterns for each cluster are available.

To determine the optimal model complexity, the RMS error (RMSE) for an increasing number of classes was computed. These experiments show that the largest gain in explained variance is obtained using the first 6 classes. The optimal number of clusters $\approx 10$

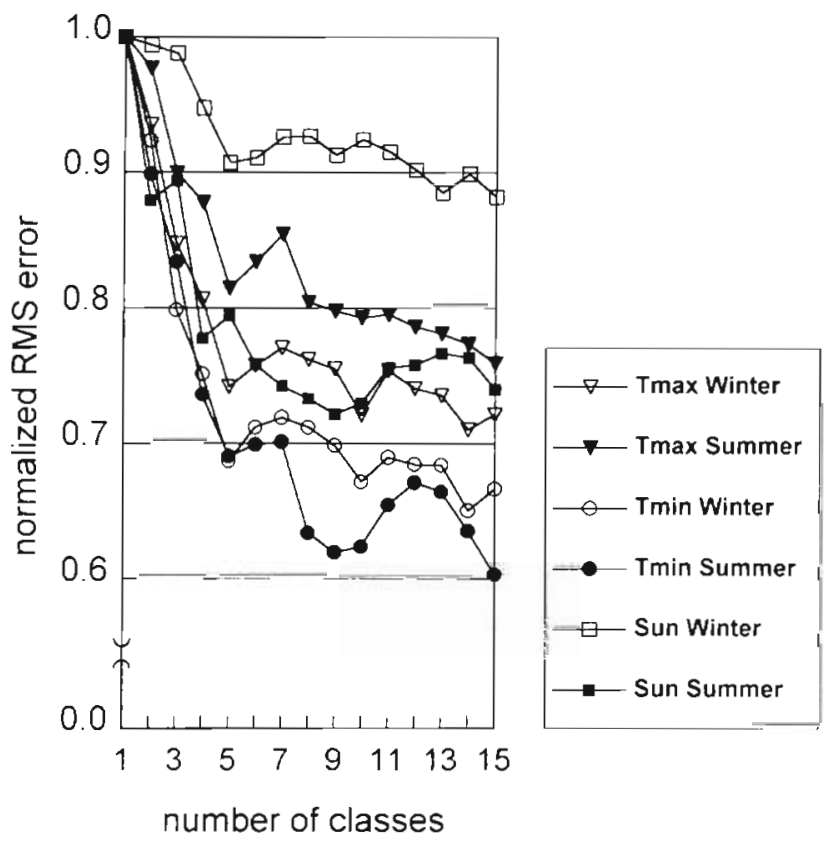

Fig. 4. Dependency of the RMSE on an increasing number of classes (weather-pattern) 
(cf. Fig. 4). An increase of this number will only result in a marginal gain of information but will lead to statistical instability and disproportionately high computational expense

\subsection{Regression}

A regression analysis following the classification is valuable for 2 reasons: (1) the information given by the mean geopotential level has not been taken into account in the classification step and (2) there are variables (see Table 1) which can be directly obtained from grid fields and which contribute significantly to the explained variance of the predictands.

A further gain in explained variance is possible by performing a multiple, conditional screening regression analysis with 39 potential predictors (Table 1), exclusively derived from upper air fields $[44$ predictors, if humidity is considered (cf. Section 4.3)]. It may seem that $\sim 40$ is quite a large number of potential predictors, but on the other hand, for the development of model output statistics (MOS) systems, several hundred potential predictors are included. The maximum number of predictors used for each predictand is, however, restricted to 4 . Increasing this number would result in only marginal improvement, and besides, overfitting can occur, in spite of cross-validation techniques used. Fewer than 4 predictors are considered if the estimation of the error averaged for both independent samples increases. The above mentioned crossvalidation was performed by dividing the data sample into 2 parts, assigning even-numbered days to subsample 1 and odd-numbered days to subsample 2 .

Note: A very important and well-known predictor is the persistence. However, if downscaling of climate model scenarios is performed, only the approximate persistence can be incorporated because the predictand of the time step $t-1$, which would be necessary, is not available. The pseudo-persistence, which is used instead, is the downscaled predictand of the previous time step.

\section{DISCUSSION OF THE RESULTS}

\subsection{Classification step}

Averaged weather patterns of the 1000 and $500 \mathrm{hPa}$ levels are immediately available after the classification step. As an example, Fig. 5a, b shows the 10 summer weather patterns for the 1000 and $500 \mathrm{hPa}$ levels with subjectively assigned pattern names [in accordance with synoptical/climatological experience and the Hess \& Brezowski (1969) and Lamb (1953) nomenclature].
Using the archive of daily surface data, grouped by climate region (cf. Section 2) and weather patterns, seasonal mean values and variance of all weather elements are computed. As an example, Fig. 6 shows the 2-dimensional distributions of summer mean values for maximum temperature and sunshine duration. Comparing these results with Fig. 5 again shows good agreement of class mean values with the given circulation patterns. The westerly pattern (WT), which occurs very frequently, displays low temperature and little sunshine within all climate regions. Class 6 (ACE), which was found on $5 \%$ of all days, accounts for extreme warm and sunny periods in Central Europe, which is in good agreement with climatological experience. It is noteworthy that, for example, the variability of sunshine duration across all classes is much larger in the coastal area than in the upper Rhine Valley. Furthermore, these effects show strong seasonality.

\subsection{Regression step}

The main goal of regression investigations was a decrease in variance of all weather elements within each circulation pattern. Table 2 shows the explained variance or reduction of variance ( $R V$, Eq. 5) according to region and weather element:

$$
R V=\left(1-\frac{M S E}{M S D}\right) \times 100
$$

where $R V=$ reduction of variance or explained variance; $M S E=$ mean square error of downscaled variable (observation - downscaled value); $M S D=$ mean squared deviation from the sample's climate (observation - annual cycle).

Clearly, the temperature regime is well interpreted ( $R V$ ranging from 75 to $81 \%$ ). Satisfactory results can be obtained for humidity, cloud cover, and sunshine duration, whereas precipitation is currently sub-optimally regionalized ( $R V$ about 20\%). However, the downscaling quality is improved if we do not investigate the amount of precipitation but instead its probability (see the last 3 columns and footnotes of Table 2). This effect is most striking in the climate regions that are less affected by orography.

Regression analysis shows, furthermore, that the frequency and importance of the predictors selected strongly depend on season, predictand, weather type and the number of predictors entered into the regression procedure. The following list displays (taking summer as an example again, and for an increasing number of predictors entered into the stepwise screening procedure) the selected predictors ordered by frequency: 
a
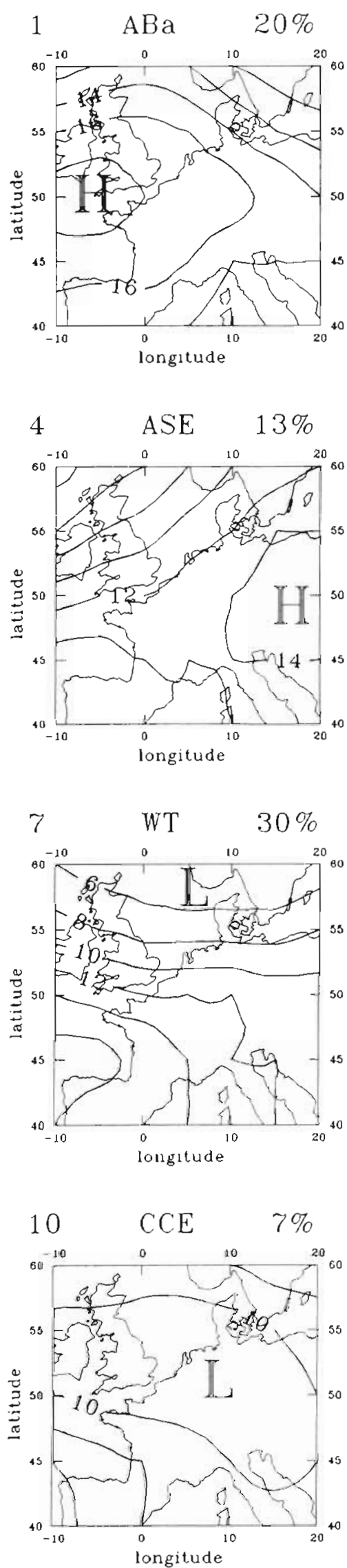
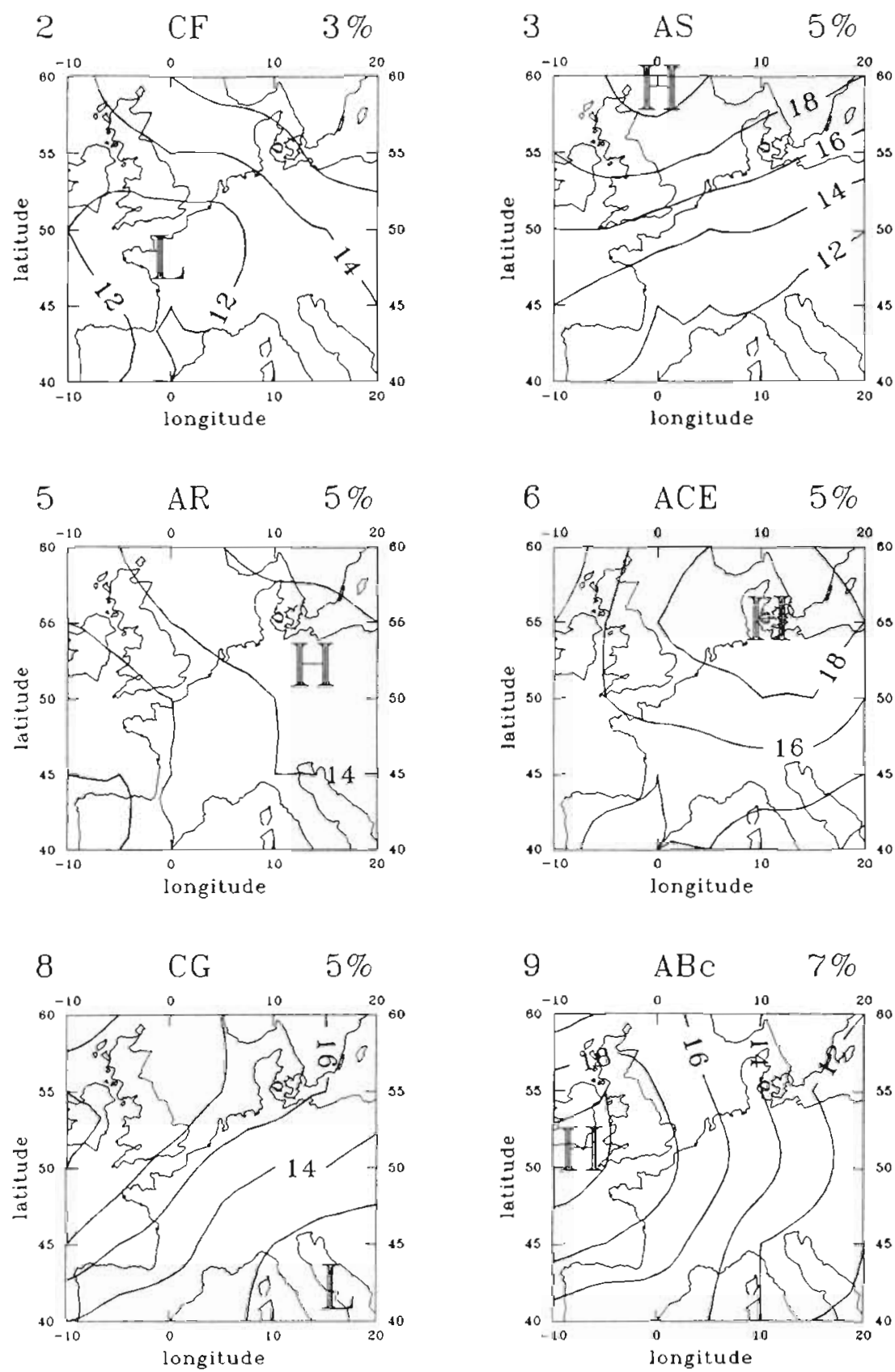

$\mathrm{ABa}$ Anticyclone British Isles, anticyclonal Central Europe

CF Cyclone France

AS Anticyclone Scandinavia

ASE Anticyclone Southern Europe

AR Anticyclonic Ridge

ACE Anticyclone Central Europe

WT Westerly Type

CG Cyclone Genova

$A B c$ Anticyclone British Isles, cyclonal Central Europe

CCE Cyclone Central Europe 
b
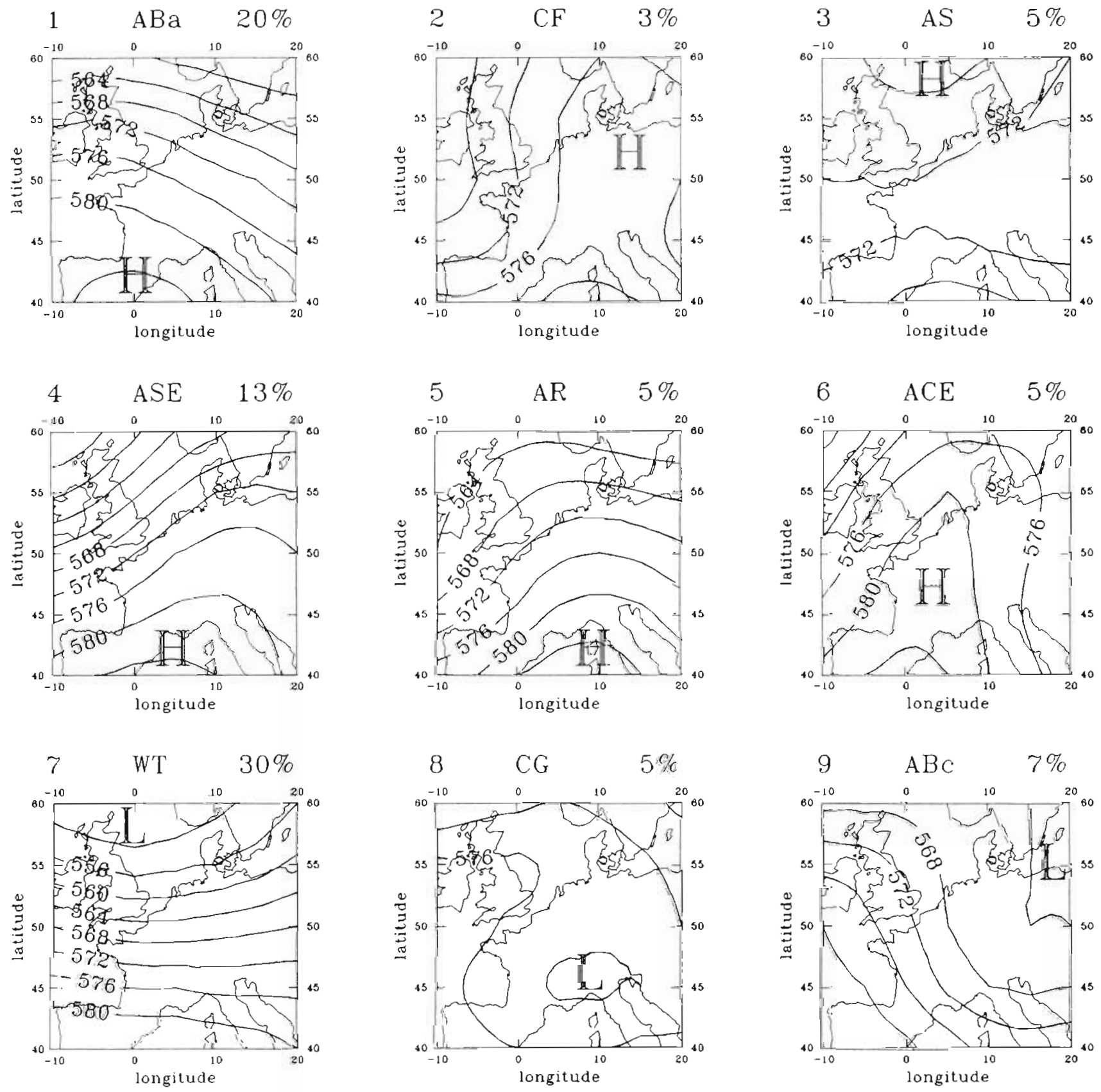

$10 \quad \mathrm{CCE} \quad 7 \%$

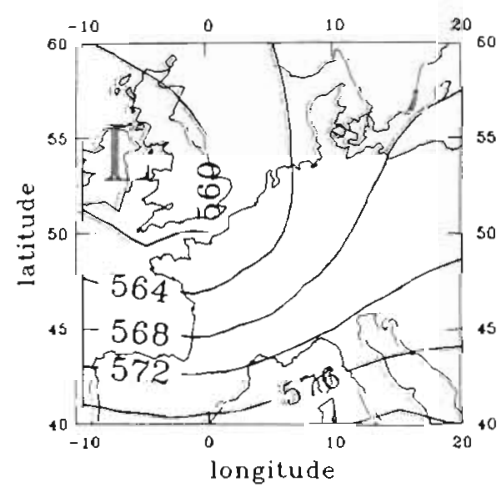

Fig. 5. Composite (a) $1000 \mathrm{hPa}$ and (b) $500 \mathrm{hPa}$ geopotential charts for summer (JJA) in Central and Western Europe. Isoline spacing is $2 \mathrm{gpm}$ in (a), and $4 \mathrm{gpm}$. in (b). Percentage values at the top right of each graph indicated the frequency of the respective weather pattern 
First step (1 predictor used):

- 500-700 hPa thickness at reference point $\mathrm{p}_{0}$

- 700 hPa vorticity

- 500-700 hPa thickness as an areal mean

- $500 \mathrm{hPa}$ vorticity, meridional component of $700-$ $1000 \mathrm{hPa}$ thermal wind, areal mean of $500-1000 \mathrm{hPa}$ thickness (all three with comparable frequency of selection).

Naturally, first predictors have the largest contribution to the explained variance. If 2,3 or 4 predictors are
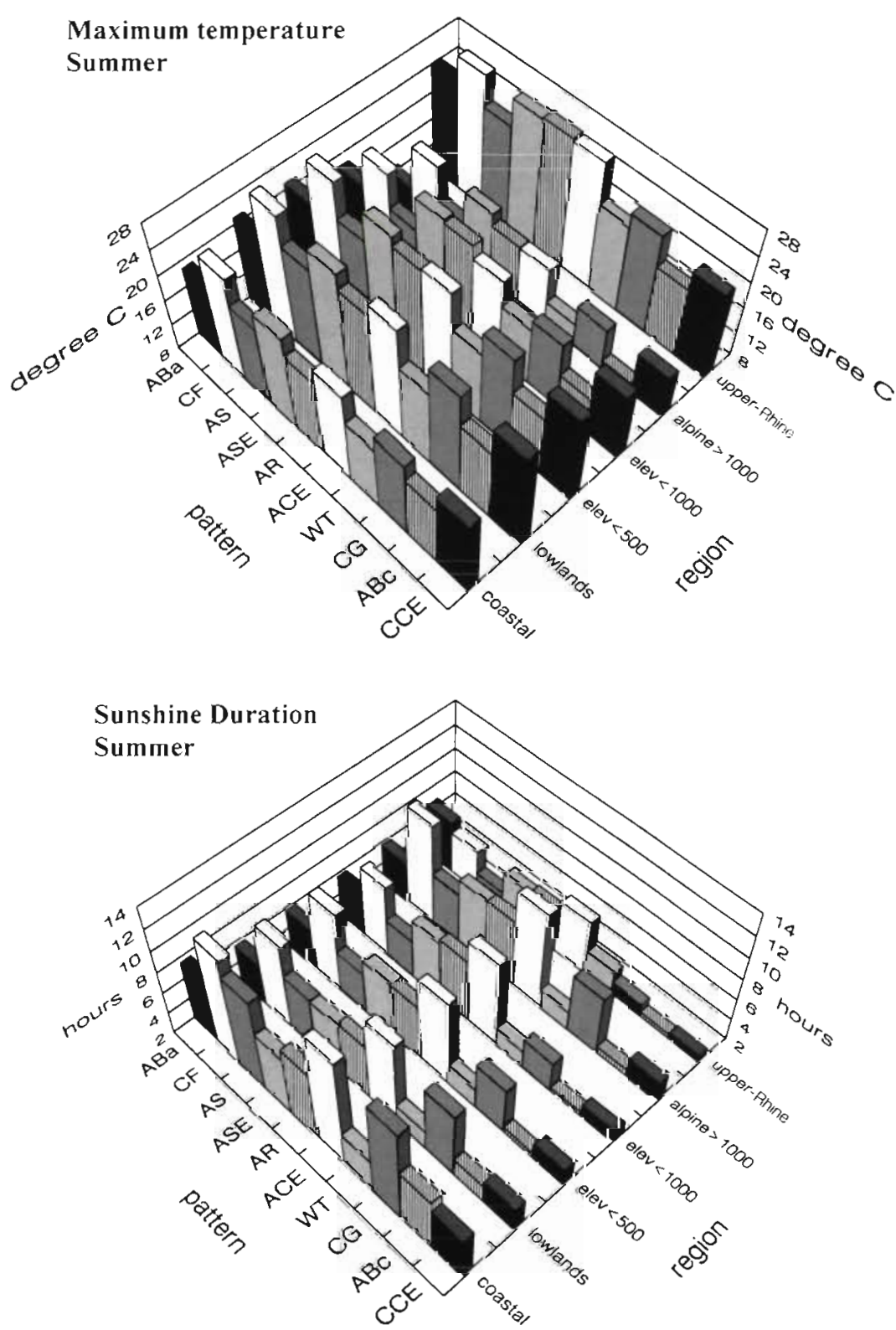

Fig. 6. Distribution of summer maximum temperature and sunshine duration as a function of weather pattern (see Fig. 5) and climate region entered into the regression, the following turn out to be of additional importance:

- zonal component of $700-1000 \mathrm{hPa}$ thermal wind

- meridional component of 700-1000 hPa thermal wind

- meridional component of $1000 \mathrm{hPa}$ geostrophic wind.

Further splitting of the results into temperature and humidity regimes shows that the most important predictor for improving the downscaling efficiency for temperature is $700-1000 \mathrm{hPa}$ thickness (see Fig. 7a), whereas regionalization of sunshine and humidity benefits most from $700 \mathrm{hPa}$ vorticity (see Fig. 7 b). Surprising as it may seem at first glance, the dominance of the $700 \mathrm{hPa}$ level in predictor selection is elucidated by the fact that this particular level was not used in the classification, and that its inherent information potential becomes effective in the regression step.

There is a remarkable seasonal dependency: thickness, for example, quite naturally is more important in summer than in winter.

\subsection{The problem of incorporating $700 \mathrm{hPa}$ humidity}

Even though we recognize the potential information to be gained from including $700 \mathrm{hPa}$ humidity fields, the classification step was not performed for 3 reasons:

(1) the daily humidity gridfields are restricted to a $17 \mathrm{yr}$ period; in this context a further subdivision into development and validation samples is not feasible

(2) the numerical analysis procedure used to obtain humidity field's was altered several times over the 17 yr period

(3) the scale of atmospheric humidity patterns may not match the scale of geopotential patterns.

Still, use of $700 \mathrm{hPa}$ humidity improves regression results. To verify this assumption the same limited data sample of 17 years was analyzed, even though, as a consequence, 
Table 2. Explained variance (\%) for several predictands in the 6 regions, derived from daily data

\begin{tabular}{|c|c|c|c|c|c|c|c|c|c|}
\hline Area & $\operatorname{Tmax}$ & Tmin & Tmean & $\mathrm{RH}$ & Cloud & Sun & Prec $[\mathrm{mm}]$ & Prec1 $^{\mathrm{a}}$ & Prec $2^{b}$ \\
\hline Coast & 74.4 & 71.0 & 77.9 & 23.9 & 48.6 & 39.3 & 16.5 & 32.6 & 23.3 \\
\hline Lowlands & 80.3 & 71.7 & 82.7 & 43.1 & 49.6 & 44.3 & 13.9 & 27.2 & 20.0 \\
\hline$<500 \mathrm{~m}$ & 81.9 & 75.4 & 84.6 & 45.9 & 47.5 & 45.7 & 14.3 & 23.1 & 18.3 \\
\hline$<1000 \mathrm{~m}$ & 76.7 & 79.4 & 79.6 & 51.2 & 41.4 & 37.9 & 22.2 & 17.6 & 16.3 \\
\hline Alpine & 78.4 & 78.1 & 80.9 & 56.4 & 58.5 & 50.9 & 25.4 & 20.8 & 16.9 \\
\hline Upper Rhine & 77.8 & 71.2 & 79.8 & 37.1 & 44.2 & 31.8 & 11.6 & 19.6 & 14.3 \\
\hline Combined areas & 78.3 & 74.5 & 80.9 & 42.9 & 48.3 & 41.7 & 20.5 & 23.5 & 18.2 \\
\hline
\end{tabular}

the statistical stability was somewhat reduced

The improvement obtained by incorporating $700 \mathrm{hPa}$ humidity for several weather elements is described by the explained variance depicted for summer in Table $3 a-c$. The main findings - also taking into account other seasons-are as follows:

- all weather elements display a great variability between circulation patterns, again depending on season and climate region

- the whole temperature regime is more sensitive in summer than in winter

- minimum temperature is far more sensitive to $700 \mathrm{hPa}$ humidity than maximum temperature (not shown here)

- summer weather patterns ACE and CG (see Fig. 5), which feature a wellpronounced northeasterly surface flow into Central Europe, are associated with high $R V$ ratios in the minimum temperature

- cyclonal weather types (patterns CF, CG and CCE) do not account for a significant improvement in $R V$ ratios, if relative humidity is incorporated

- for the sunshine duration, best results are obtained for patterns AS, AR and ABC

- the elevated areas below $500 \mathrm{~m}$ (area 3) seem to respond well, if minimum temperature, surface humidity, and sunshine are analyzed; the improvement in maximum temperature is remarkably unpronounced.

The seeming paradox of negative values of $R V$ (incorporating the additional information of the humidity fields worsens the results) must be explained by the screening procedure used. Searching for maximum improvement is performed on a 'downhill' basis: The predictor with the highest $R V$ is determined; then the predictor with the highest $R V$ in
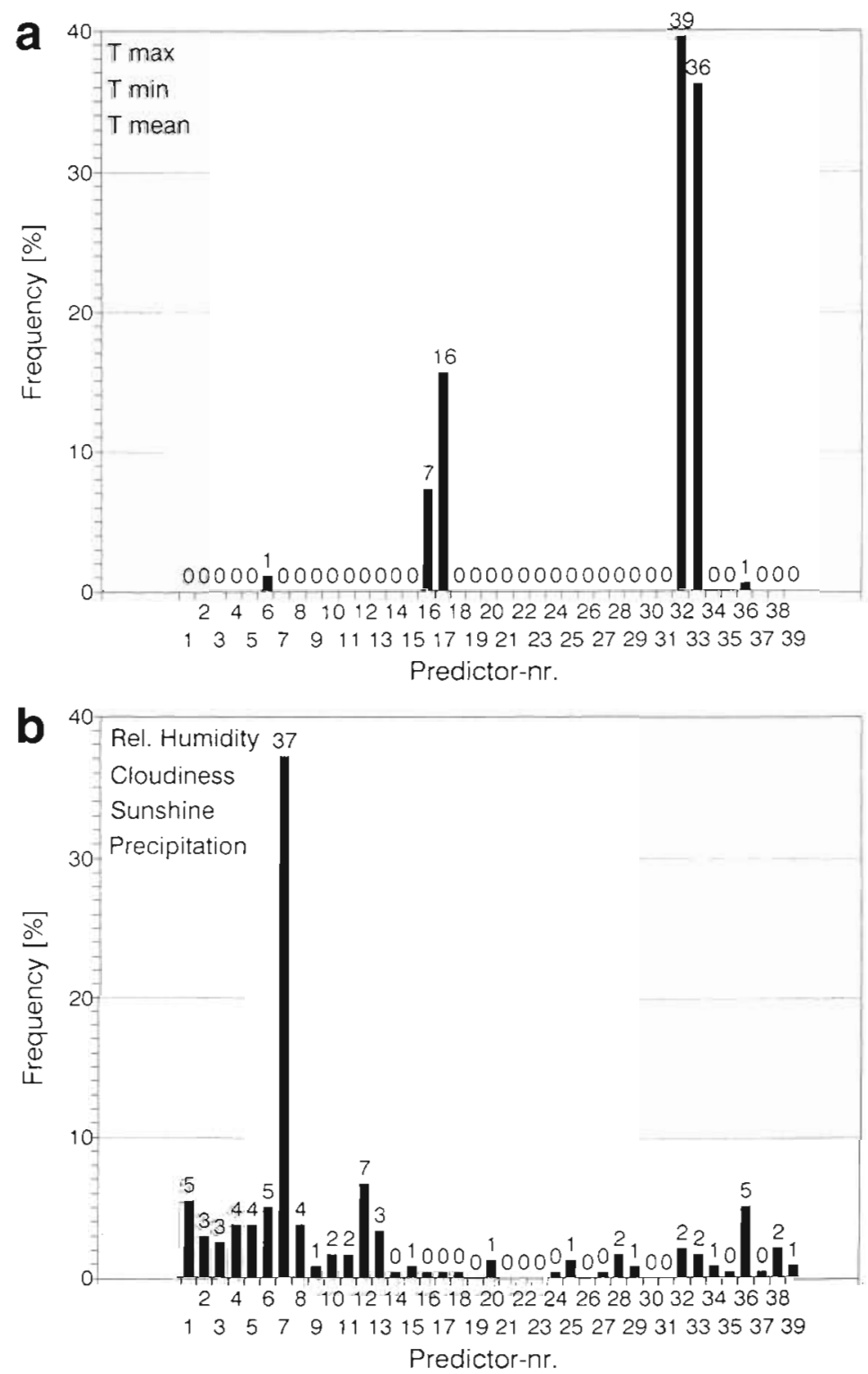

Fig. 7. Results of a screening regression analysis. (a) Temperature regime (Tmax, Tmin, Tmean); only 1 predictor entered. (b) As in (a), but for the humidity/sunshine regime (relative humidity, cloudiness, sunshine, precipitation). Predictors are listed in Table 1 
Table 3. Gain in explained by incorporating $700 \mathrm{hPa}$ humidity (in \%), calculated as $\frac{\text { RMSE(with humidity) }}{\text { RMSE(without humidity) }} \times 100$. Data basis: summer 1966-1993. (-) indicates a gain in explained variance between 0.1 and -0.1

\begin{tabular}{|c|c|c|c|c|c|c|c|c|c|c|c|}
\hline Pattern frequency $(\%)$ : & $\begin{array}{c}\mathrm{ABa} \\
20\end{array}$ & $\begin{array}{c}C F \\
3\end{array}$ & $\begin{array}{c}\text { AS } \\
\mathbf{5}\end{array}$ & $\begin{array}{c}\text { ASE } \\
\mathbf{1 3}\end{array}$ & $\begin{array}{c}\mathrm{AR} \\
5\end{array}$ & $\begin{array}{c}\mathrm{ACE} \\
5\end{array}$ & $\begin{array}{c}\text { WT } \\
30\end{array}$ & $\begin{array}{c}\text { CG } \\
5\end{array}$ & $\begin{array}{c}A B C \\
7\end{array}$ & $\begin{array}{c}C C E \\
7\end{array}$ & Mean \\
\hline \multicolumn{12}{|l|}{ (a) Minimum temperature } \\
\hline Coast & - & - & - & - & 7.6 & - & 3.6 & 10.6 & - & - & 1.9 \\
\hline Lowlands & 2.7 & - & 3.7 & - & 2.3 & 4.3 & 1.5 & 36.7 & 17.1 & - & 4.5 \\
\hline Elevation $<500$ & 12.6 & 14.5 & 3.2 & 7.1 & 6.0 & 33.3 & 4.3 & 33.5 & 13.7 & - & 10.0 \\
\hline Elevation $<1000$ & 10.0 & - & 1.3 & - & - & - & 5.8 & 13.3 & 2.0 & - & 4.5 \\
\hline Alpine $>1000$ & - & - & 1.8 & - & - & - & 1.4 & 10.3 & 2.9 & - & 1.2 \\
\hline Upper Rhine & 4.3 & 5.6 & - & 2.2 & 13.5 & 20.9 & 6.7 & 8.2 & 8.9 & 2.9 & 6.3 \\
\hline Mean & 4.9 & 3.3 & 1.5 & 1.5 & 4.9 & 9.7 & 3.9 & 18.8 & 7.4 & 0.5 & 4.7 \\
\hline \multicolumn{12}{|l|}{ (b) Relative humidity } \\
\hline Coast & 13.6 & 9.9 & 25.0 & 20.8 & 23.8 & 20.7 & 6.5 & 7.7 & 10.0 & - & 12.2 \\
\hline Lowlands & 5.0 & 2.3 & 29.8 & 14.4 & 7.3 & 10.4 & 6.8 & 4.1 & 23.6 & - & 9.3 \\
\hline Elevation $<500$ & 7.9 & -13.0 & 12.0 & 14.0 & 15.6 & 21.7 & 17.9 & 8.1 & 11.4 & - & 12.0 \\
\hline Elevation $<1000$ & -0.3 & 5.0 & 4.6 & 7.4 & 7.2 & 28.8 & 7.7 & - & 7.7 & - & 6.0 \\
\hline Alpine $>1000$ & 3.0 & - & 17.5 & 4.2 & 3.6 & 24.1 & 12.5 & - & 0.2 & 1.4 & 7.4 \\
\hline Upper Rhine & 9.3 & - & -6.3 & 2.9 & 10.0 & 21.9 & 7.3 & - & 17.3 & 1.7 & 7.1 \\
\hline Mean & 6.4 & 0.7 & 13.8 & 10.6 & 11.2 & 21.3 & 9.8 & 3.3 & 11.7 & 0.6 & 9.0 \\
\hline \multicolumn{12}{|l|}{ (c) Sunshine duration } \\
\hline Coast & - & 8.1 & 24.4 & 6.2 & 27.9 & - & - & 12.1 & 8.3 & - & 4.8 \\
\hline Lowlands & 0.7 & 3.2 & 28.1 & 2.4 & 26.3 & 5.6 & - & 4.9 & 15.4 & - & 4.9 \\
\hline Elevation $<500$ & 2.6 & 8.6 & 16.2 & 5.6 & 30.4 & 7.0 & 14.6 & 12.5 & 13.5 & - & 10.1 \\
\hline Elevation $<1000$ & 2.4 & - & - & 3.0 & 17.9 & -5.2 & 10.2 & 16.6 & 22.8 & - & 7.0 \\
\hline Alpine $>1000$ & 5.2 & 4.5 & 11.2 & -0.7 & - & -0.9 & 11.2 & 6.5 & - & 3.5 & 5.6 \\
\hline Upper Rhine & 7.5 & - & - & 0.6 & 6.6 & 20.3 & 6.3 & 1.2 & 16.5 & 8.8 & 6.7 \\
\hline Mean & 3.1 & 4.1 & 13.3 & 2.8 & 18.2 & 4.5 & 7.0 & 9.0 & 12.8 & 2.1 & 6.5 \\
\hline
\end{tabular}

combination with those from the preceding step(s) is determined (which reduces the number of combinations), so that it cannot be guaranteed that the absolute best combination of predictors is found. It may be that the 'path' taken while selecting up to 4 predictors leads to a higher $R V$ if humidity is not used in the regression. Table 3 illustrates that this effect (1) rarely occurs and (2) is basically of lesser magnitude.

\subsection{Reconstruction of seasonal averages}

Seasonal averages were computed from daily modelled and observed data (temperature, sunshine, precipitation, etc.). To obtain modelled values, daily grid fields were assigned to their most similar circulation patterns (cf. Section 3.1). For each pattern and in each region, the predictors from the screening analysis were applied in the regression modelling (cf. Section 3.2).

Furthermore, to ensure the stability of the downscaling method, the cross validation as described in Section 3.2 was performed for the regression step. The whole data sample was additionally divided into a development (1967-1983) and a validation (1984-1992) sample. The comparison between observed and modelled seasonal averages is shown in Fig. 8a (maximum tem- perature) and Fig. $8 \mathrm{~b}$ (sunshine duration) for the lowlands of northern Germany. Explanations for the dotted lines (control run results) in these graphs are given in Section 5. It is evident that the correlation between both curves, also shown in the plot, is highest for temperature in winter (DJF) and summer (JJA), whereas the transitional seasons spring (MAM) and autumn (SON) show a less developed linkage $(r<0.80)$, which is in good accordance with synoptic experience.

For sunshine duration (Fig. 8b) it must be stated that the correlation strongly depends on this weather variable's absolute level. For spring and summer the high interannual variability is well described (correlations $r=0.63$ and 0.72 ). For autumn and winter seasons which exhibit very low interannual variability, no significant correlations were found. Table 4 gives an overview for area 2 (lowlands) of the correlation between observed and modelled seasonal averages for all weather elements investigated. Generally, the temperature regime is well explained. Relative humidity, which is determined from daily averaged observations, shows much smaller correlations, whereas precipitation - at least for this region-is also modelled well. Comparable results are obtained if daily data instead of seasonal averages are considered (cf. Table 2). 
a
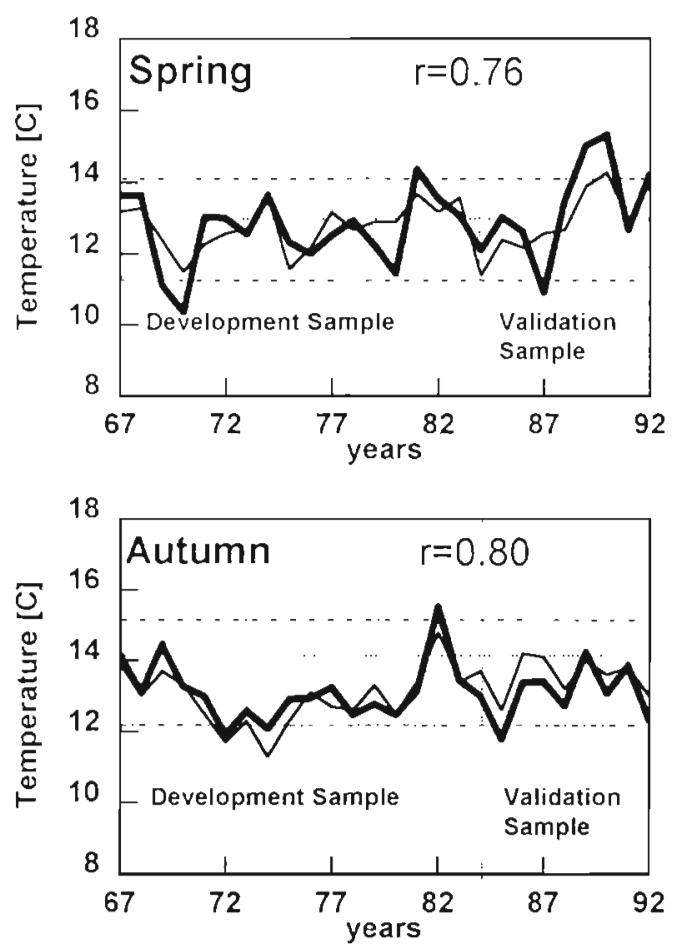

b
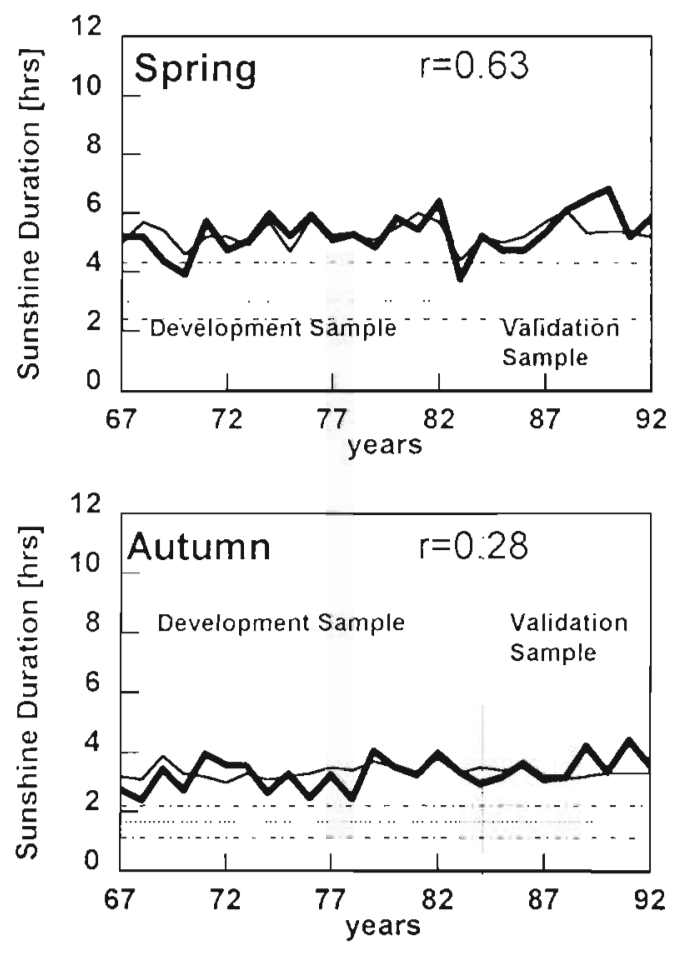

- Obs Model C591
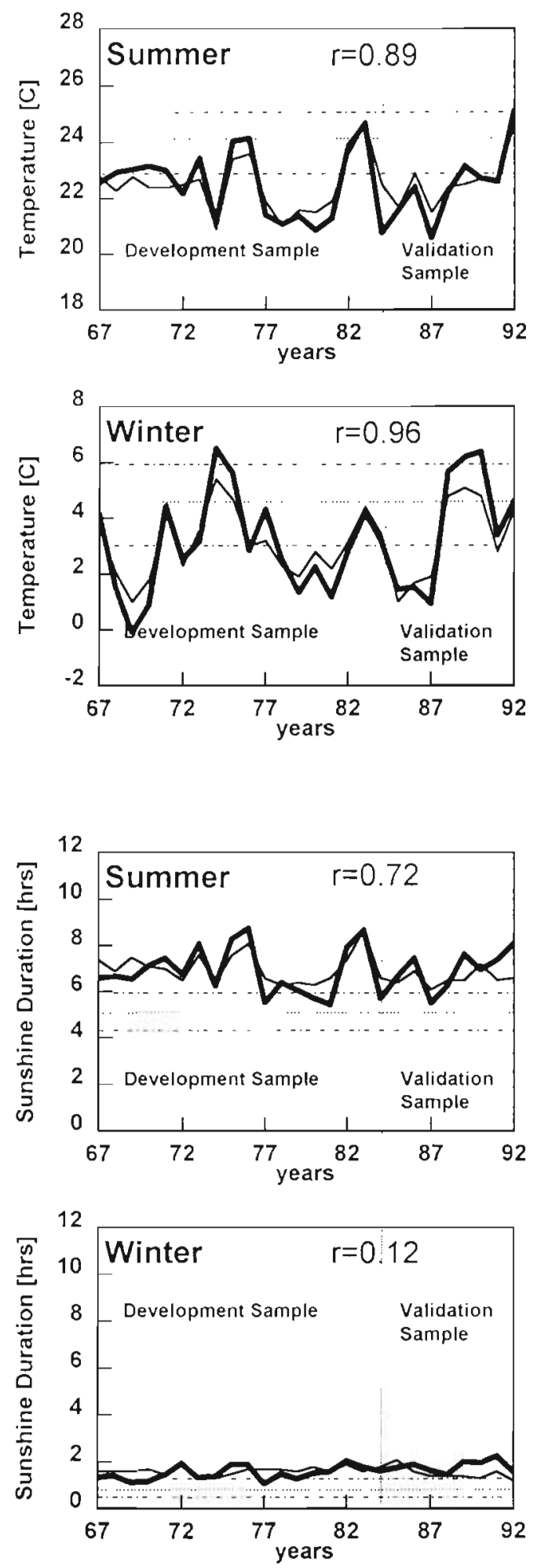

upper range $\cdots$..... lower range

Fig. 8. (a) Seasonal averages of observed and modelled maximum temperature (continuous lines) based on climate data and temperature means and ranges (dotted lines) from a 13 yr GCM control run for area 2 (lowlands). Modelling is based on a development sample (1967-1983). Correlation coefficients are given in the upper part of each graph; cf. Table 4. (b) as in (a), but for 
The validity of the method is underscored by the fact that both curves in Fig. 8 are close even in the validation period. Although the data sample is comparatively short, it can be expected that the obvious good fit found in the validation sample should make the method suitable for predicting long-term trends and downscaling GCM scenarios, as well
Table 4 . Correlation between observed and modelled seasonal time series, 1967-1992. Lowlands region. Development sample: 1967-1983. Validation sample: 1984-1992. Significant correlations are in bold type; cf. Fig. 8

\begin{tabular}{|lccccccc|}
\hline & Tmax & Tmin & Tmean & RH & Cloud & Sun & Prec \\
\hline Spring &. $\mathbf{7 6}$ & .82 & .83 & .11 & .73 & .63 & .55 \\
Summer & .89 & .88 & .92 & .27 & .71 & .72 & .57 \\
Autumn & .80 & .80 & .76 & .17 & .67 & .28 & .51 \\
Winter & .96 & .93 & .95 & .67 & .30 & 12 & .41 \\
\hline
\end{tabular}

\section{DOWNSCALING RESULTS FROM A GCM CONTROL RUN}

In the preceding sections, a method was introduced which aims to analyze climate model results. Encouragcd by plausible results 'along the way', as a first application, data from Control Run 591 of the MPI (Max Planck Institute of Meteorology, Hamburg) ECHAM4 model were used to show the method's validity.

Control Run 591 (C591) is an experiment which attempts to reconstruct the current climate conditions using ECHAM4 at T42 resolution. Sea-surface temperature and sea-ice extent were prescribed using observations. Thirteen years of daily gridded fields $\left(5^{\circ} \times 5^{\circ}\right.$ resolution) were extracted from the archives of the DKRZ (Deutsches Klimarechenzentrum).

By analyzing the C591 fields (using the classification step described in Section 3.1) and comparing them with the 1966-1993 climatology it turned out that the dominant circulation pattern (WT) was overrepresented in the control run, but the frequency distribution was reproduced qualitatively. Consequently, a discrepancy between the climate data and the downscaled GCM simulation appears. The mean level and absolute range of the $13 \mathrm{yr}$ GCM simulation, which are given in Fig. 8 (dotted lines), show a satisfactory reproduction of the temperature regime in spring and autumn. On the other hand, in summer and winter, neither the range nor the average is in agreement with climatology. This may be partly explained by the above mentioned dominance of moist air masses advected in WT circulation, which is confirmed by the fact that for each season the sunshine duration (Fig. 8b) of the control run is generally below the climatological average. It should be noted that the variability in each season is comparable for both sources.

\section{CONCLUDING REMARKS}

It can be expected that the method presented and applied here will be a useful tool for downscaling large-scale climate model outputs into regional, as well as local, weather elements. This is particularly valuable if model scenarios are analyzed.

Furthermore, from the pool of available downscaling procedures (statistical, dynamical, and combined methods) this particular method is very economical; it does not require major computer resources and can be transferred to different climate zones.

The ability to interpret results obtained from applying this method is of course subject to the quality of input data given by the climate models. One of the requirements of this method is that the causality between weather patterns and surface weather elements is of equal type in the learning sample (observations) and in the future. Changes in these relationships may be caused by (1) changes within the climate system or (2) inadequate reproduction of present and future circulation patterns in climate models (von Storch 1995). This is, of course, a general drawback of all statistical prediction methods.

Returning to the central issue of this paper, it is clear that this method is basically successful. It is especially successful for downscaling the temperature regime, and less successful for the humidity, cloud cover and sunshine duration regimes. However, much work still needs to be done to improve the downscaling of a weather element of crucial importance: precipitation.

A first application to GCM control run data showed that downscaled climate was in good agreement with the observed climate. Deviations may be explained by an inadequately reproduced circulation regime, especially by the overrepresentation of zonal circulation types.

Acknowledgements. This work was sponsored by grant 07VKV01/1-30 of the German Ministry for Education, Research, Science, and Technology (BMBF) as a part of its research programme 'Climate Variability and Signal Detection'

\section{LITERATURE CITED}

Balzer K (1994) On the 'state-of-the-art' in local weather forecasting. Met Apps 1.121-128 
Bardossy A, Duckstein L, Bogardi I (1995) Fuzzy rule-based classification of atmospheric circulation patterns. Int $\mathrm{J}$ Climatol 15:1087-1097

Burrows WR, Benjamin M, Beauchamp S, Lord ER, McCollor D. Thomson B (1995) CART decision-tree statistical analysis and prediction of summer season maximum surface ozone for Vancouver, Montreal and Atlantic regions of Canada. J Appl Meteorol 34:1848-1862

Chen JM, Harr PA (1992) On the interpretation of extended empirical orthogonal function analysis. 5th International Meeting on Statistical Climatology, 22-26 June 1992, Toronto, Canada, p 237-240

Enke W (1986) Ein Verfahren zur Aufstellung statistischer Prognosealgorithmen als Einheit von Cluster- Diskriminanz- und Regressionsanalyse. Z Meteorol 36(2): $117-122$

Enke W (1994) The extreme summer in 1992 over northern Germany. In: Schellnhuber HJ, Enke W, Flechsig M (eds) PIK Reports, Vol 4, No. 2

Enke W, Spekat A (1995) A two-step method to transform climate model outputs into regional and local weather elements. 6th International Meeting on Statistical Climatology, 19-23 June 1995, Galway, Ireland, p 339-342

Forgy EW (1965) Cluster analysis of multivariate data: efficiency versus interpretation of classifications. Biometrics 21:768-769

Frey-Buness A, Heimann D, Sausen R (1995) A statisticaldynamical downscaling procedure for global climate simulations. Theor Appl Climatol 50:117-131

Giorgi $F(1990)$ Simulation of regional climate, using a limited area model nested in a general circulation model. J

Editorial responsibility: Hans von Storch, Geesthacht, Germany
Clim 3:941-963

Giorgi F, Mearns LO (1991) Approaches to the simulations of regional climate change: a review. Rev Geophys 29: $191-216$

Hess P, Brezowsky H (1969) Katalog der Großwetterlagen Europas. Ber Dtsch Wetterdienst, Offenbach, Vol 15, Rep 113

Jessup RG, Burrows WR (1996) Neural network post processing of CART regression output. 13th Conference on Probability and Statistics in the Atmospheric Science, 21-23 February, 1996, San Francisco. American Meteorological Society, Boston

Klein WH, Lewis BM, Enger I (1959) Objective prediction of five-day mean temperature during winter. J Meteorol 16:672-682

Kutzbach J (1967) Empirical eigenvectors of sea level pressure, surface temperature, and precipitation complexes over North America. J Appl Meteorol 6:791-802

Lamb HH (1953) British weather around the year-I. Weather 8:131-136

Lamb HH (1972) Climate, present, past and future, Vol 1. Methuen, London

Mucha HJ (1992) Clusteranalyse mit Mikrocomputern. Akademie Verlag, Berlin

von Storch H (1995) Inconsistencies at the interface of climate impact studies and global climate research. Meteorol Z NF $4: 72-80$

Zorita E, Hughes JP, Lettenmaier DP, von Storch H (1995) Stochastic characterization of regional circulation patterns for climate model diagnosis and estimation of local precipitation. J Clim 8:1023-1042

Manuscript first received: October 24, 1995

Revised version accepted: November 18, 1996 